\title{
TRAVAILLER SUR LA GUERRE D'INDÉPENDANCE ALGÉRIENNE : BILAN D'UNE EXPÉRIENCE HISTORIENNE
}

\author{
Sylvie Thénault
}

Verdier | « Afrique \& histoire »

2004/1 vol. 2 | pages 193 à 209

ISSN 1764-1977

ISBN 2-86432-399-0

Article disponible en ligne à l'adresse :

https://www.cairn.info/revue-afrique-et-histoire-2004-1-page-193.htm

Distribution électronique Cairn.info pour Verdier.

(C) Verdier. Tous droits réservés pour tous pays.

La reproduction ou représentation de cet article, notamment par photocopie, n'est autorisée que dans les limites des conditions générales d'utilisation du site ou, le cas échéant, des conditions générales de la licence souscrite par votre établissement. Toute autre reproduction ou représentation, en tout ou partie, sous quelque forme et de quelque manière que ce soit, est interdite sauf accord préalable et écrit de l'éditeur, en dehors des cas prévus par la législation en vigueur en France. Il est précisé que son stockage dans une base de données est également interdit. 


\title{
Travailler sur la guerre d'indépendance algérienNe: BILAN D'UNE EXPÉRIENCE HISTORIENNE
}

\author{
Sylvie Thénault
}

Cet article se propose de revenir sur l'écriture de l'histoire de la guerre dite " guerre d'Algérie " dans le contexte des années 1990-2000. En effet, ces années ont vu l'avènement d'une nouvelle génération d'historiens, qui n'ont pas vécu cette guerre, et qui y réinvestissent, en réalité, les enjeux politiques de leur propre époque. L'ouverture des archives du Service historique de l'armée de terre (SHAT), entre 1992 et 2000, a également influencé I'historiographie, en suscitant de multiples travaux sur ses aspects militaires. Elle a cependant aussi donné lieu à une histoire reflétant le point de vue militaire, que d'autres sources devraient corriger. Enfin, les échanges scientifiques avec l'Algérie, longtemps marqués par l'ancien rapport colonial, sont aujourd'hui également perturbés par l'absence de démocratie et la guerre actuelle dans ce pays. Ces conditions invitent à redéfinir la démarche des historiens travaillant sur la guerre d'indépendance algérienne.

En décembre 2000, la coïncidence d'une polémique et de la soutenance d'une thèse sur la torture propulsait l'histoire de la guerre d'indépendance algérienne hors du champ scientifique, dans une arène politico-médiatique prompte à voir du neuf dans un événement banal pour les milieux universitaires ${ }^{\mathrm{I}}$. Un fossé séparant d'ordinaire production historique et médias, ces derniers ont vu une révélation soudaine dans l'achèvement d'un travail mené depuis plusieurs années, dans l'isolement et les tourments propres au parcours initiatique du doctorant. Y associant une thèse sur la justice soutenue un an plus tôt, à laquelle fut bientôt ajoutée une troisième consacrée aux appelés et publiée depuis 1998, ils ont construit une figure incarnant le renouveau de cette histoire: celle de trois " jeunes femmes intrépides et obstinées » brisant les silences coupables de la France contemporaine ${ }^{2}$. Jolie construction littéraire, esthétique, cette expression a été abondamment reprise. Sa

Chargée de recherche au CNRS (Institut d'histoire du temps présent, Cachan, France), Sylvie ThÉNault est l'auteur de Une drôle de justice. Les magistrats dans la guerre d'Algérie (Paris, 2001).

I. Il s'agit de la thèse de Raphaëlle Branche (R. Branche, 20OI), dirigée par Jean-François Sirinelli.

2. Il s'agit de ma thèse (S. ThÉNAult, 20OI), dirigée par Jean-Jacques BeCKer, et de celle de Claire MaussCopeaux (C. Mauss-Copeaux, 1998), dirigée par Annie Rey-Goldzeiguer. L'expression " trois jeunes femmes intrépides et obstinées" est de Robert Bonnaud (2000 : p. 7I). 
signification plaisait aussi, l'effet de redondance créé par l'association des deux adjectifs soulignant particulièrement les obstacles qu'il aurait fallu surmonter, la jeunesse et le sexe féminin s'ajoutant au tabou et augmentant d'autant l'exploit.

Pourtant, il a semblé facile de faire une thèse sur cette guerre ; plus facile, en tout cas, de travailler dans le silence et l'indifférence des années i99o que dans le bruit et la polémique des années 2000-200I. Paradoxalement, le tabou, plus supposé que réel, d'ailleurs, est moins un handicap que sa levée proclamée, car il protège le chercheur de l'irruption des journalistes, des militants, des témoins... dans son champ. Henry Rousso explique ainsi le malaise ressenti : "Loin de rencontrer une résistance soutenue, les historiens allaient devoir affronter au contraire la mise au présent progressive de ce passé, quittant peu à peu leur rôle d'observateurs pour devenir, avec d'autres, les acteurs de cette anamnèse ${ }^{3}$. Cette mutation perturbe en effet le chercheur concentré sur sa tâche, entre ses archives, ses livres, ses revues, les soutenances, les séminaires et les colloques. Il est tentant, alors, d'ignorer l'extérieur, de balayer par l'ironie l'idée d'un renouveau de l'historiographie de la guerre d'indépendance algérienne, au motif qu'elle a été éclairée par une coïncidence, soulignée par les médias et désignée par une de ces formules percutantes qu'ils affectionnent; il est tentant, aussi, de répondre que les historiens ont travaillé et consulté des archives depuis longtemps.

Cependant, c'est une nouvelle génération qui émerge, celle qui n’a pas connu la guerre et qui a bénéficié de l'ouverture des archives du Service historique de l'armée de terre (SHAT). Pour Claude Liauzu, depuis les années 1950-1960, "l'histoire française de la colonisation » connaît aujourd'hui " un troisième moment dans le débat public et dans la recherche ", où, loin des "dénonciations anciennes ", il s'agit "d'ouvrir de nouvelles pistes d'investigation pour traiter un passé qui, comme d'autres, ne "passe pas" ${ }^{4}$ ". Après l'anamnèse viendrait le temps du traitement. La métaphore fait de l'événement une source de pathologie gagnant le corps social, que l'historien pourrait aider à guérir, quarante ans après. Il faudrait attendre une nouvelle génération, non marquée par la guerre, pour que s'élabore une approche de l'événement débarrassée de culpabilité.

Qu'en est-il ? Nouvelle génération, nouvelles archives, nouvelle configuration politique dans l'Algérie indépendante re-basculant dans la guerre: comment l'histoire de l'indépendance algérienne est-elle écrite en France dans les années 19902000 ? Sans chercher à dresser un état des lieux prétendant à l'exhaustivité, il s'agit ici d'ouvrir la réflexion à partir d'une expérience personnelle, car c'est aussi dans la pratique quotidienne et le cheminement individuel des historiens que se fait l'histoire. 


\section{UN RENOUVEAU DES SUJETS DE THÈSES}

L'analyse des sujets de thèse permet de mesurer le degré de légitimation d'une période historique comme objet de recherche. Elle intègre ainsi la catégorie des sujets acceptés pour évaluer la qualité d'un postulant à l'entrée dans la corporation historienne. Ce type de sujet doit en effet répondre à des critères exigeants de faisabilité, mais aussi de neutralité, pour éviter d'handicaper le déroulement postérieur de la carrière du jeune docteur, dont le travail risquerait d'être ramené à un travail journalistique ou militant. Les professeurs acceptant de diriger une thèse sur la dénommée " guerre d'Algérie " ont ainsi validé personnellement, par leur geste, cette période comme objet de recherche scientifique, en dépit des réticences que pouvait susciter un sujet d'histoire récente et, qui plus est, source de controverses. De la part de l'étudiant, le choix d'un tel sujet témoigne d'une approche décomplexée de la période, que l'environnement historiographique contribue à expliquer.

Dans les années 1960-1970, en effet, la définition des sujets de thèse relatifs à l'Algérie excluait la guerre d'indépendance, les années I9I4-I9I8 semblant constituer le terme le plus raisonnable, à un moment où l'histoire des périodes les plus proches suscitait la méfiance. La guerre d'indépendance algérienne, alors, ne faisait pas exception à la règle écartant ces périodes du domaine de l'histoire pour considérer que leur traitement relevait du journalismes. Annie Rey-Goldzeiguer et Jacques Frémeaux se sont ainsi intéressés au XIX siècle, tandis que Charles-Robert Ageron et Gilbert Meynier ont conduit leurs sujets jusqu'à l'immédiat aprèsguerre $^{6}$. À partir des années 1980 ont abouti des travaux qui, fait nouveau, incluaient la guerre, mais dans la longue durée: Guy Pervillé a ainsi travaillé sur Les étudiants algériens de l'université française (I882-1962), tandis que Benjamin Stora a étudié la biographie de Messali Hadj de I898 à 1974, avant de se consacrer à l'immigration algérienne en France, de 1922 à $1962^{7}$. Dans les années 1990, ensuite, sont parus les travaux d'Omar Carlier sur l'« histoire sociale des radicalismes algériens ", dont certains concernent le présent, et la thèse de Daniel Lefeuvre, sur l'histoire économique de l'Algérie de I830 à $1962^{\circ}$.

L'évolution se dessine donc dans les années 1980 car la période de la guerre est apparue dans la définition des bornes chronologiques des sujets de thèse. L'ouverture des archives du SHAT en 1992 est venue la renforcer. Elle a accéléré un

5. Comme le souligne Jean-Jacques Becker, "cette position était assez récente ", car, pour " la période I9I4-I9I8, le doyen Renouvin publie dès les années 20, sur des événements qu'il a vécus lui-même ». Le " refus " serait venu de la génération suivante; J.-J. BECKER (2002:38).

6. Cf. C.R. Ageron (I 97I) ; A. Rey-Goldzeiguer (i977); J. Frémeaux (I993, mais sa thèse a été soutenue en 1977); G. Meynier (I98I).

7. G. Pervillé (I984) et B. Stora, (I986 et I992).

8. O. Carlier (I995) et D. Lefeuvre (I997). 
mouvement amorcé au fur et à mesure que le temps s'écoulait, pour aboutir à un véritable tournant dans les années 1990 avec la conduite de thèses se concentrant sur la guerre elle-même et fondées sur les archives militaires. Ici encore, cette guerre reflète une évolution plus générale: en raccourcissant le temps accordé pour faire une thèse, le nouveau régime de doctorat a très certainement contribué au resserrement chronologique sur les huit années de guerre. La thèse de Djamila Amrane, consacrée aux Femmes algériennes dans la guerre, soutenue en 1988, se fondait ainsi uniquement sur des témoignages, mais se concentrait déjà sur la seule période $1954-1962^{\circ}$. Le travail de Claire Mauss-Copeaux, ensuite, pourrait être considéré comme un travail de transition: alors qu'elle a commencé avant 1992 par des entretiens, source principale de sa thèse, elle les a complétés par les archives du $\mathrm{SHAT}^{\mathrm{I}}$. Ainsi enracinée dans l'histoire longue des thèses consacrées à l'Algérie, la soutenance de thèses sur la guerre d'indépendance algérienne en 1999 et 2000 perd son originalité pour apparaitre comme le simple aboutissement d'une évolution voyant se resserrer le champ chronologique étudié et s'ouvrir de nouvelles archives.

Au moment même de la définition du sujet, l'existence des thèses précédentes créait un environnement favorable au choix de travailler sur cette guerre, car leurs auteurs avaient accédé à la notoriété et ils tenaient des séminaires, lieux de rencontres, d'échanges, de réflexions collectives. À leurs thèses s'ajoutait en outre l'ensemble de la production historique, autour notamment de l'IHTP avec Jean-Pierre Rioux et Charles-Robert Ageron: les grands colloques comme La guerre d'Algérie et les Français ", ou son pendant La guerre d'Algérie et les Algériens ${ }^{12}$; les études sur l'opinion comme Les chrétiens dans la guerre d'Algérie ${ }^{13}$ ou Les intellectuels et la guerre d'Algérie ${ }^{14}$; les études des grands acteurs ayant contesté la guerre ou soutenu le FLN comme Face à la raison d'État ${ }^{15}$ ou Les porteurs de valise ${ }^{16}$. Bernard Droz, Evelyne Lever et Benjamin Stora, de leur côté, permettaient aux débutants de s'initier à la guerre par des ouvrages de synthèse ${ }^{17}$. L'ensemble témoignait du dynamisme des historiens sur la période et rendait possible, pour un étudiant, l'idée de proposer un sujet de thèse s'y intéressant. Au début des années 1990, les étudiants arrivés à ce stade dans leur parcours universitaire appartenaient à une nouvelle génération, celle qui n'a pas connu la guerre.

\footnotetext{
9. D. Amrane (I99i).

io. C. Mauss-Copeaux (1998).

II. J.-P. Rioux (dir.) (1990).

I2. C.R. Ageron (dir.) (I997).

I3. F. BÉdARida et E. Fouilloux (dir.) (I988).

I4. J.-P. Rioux et J.-F. Sirinelli (dir.) (I99I).

I5. P. Vidal-Naquet (1989).

I6. H. Hamon et P. Rotman (1982 pour la réédition de leur travail paru en 1979).

I7. B. Droz et E. Lever (I982) et B. Stora (I993).
} 


\section{UN FOSSÉ GÉNÉRATIONNEL?}

Cette génération née après 1962, cependant, a rompu avec la façon d'aborder la question par les générations précédentes. Pour ceux qui ont toujours connu l'Algérie indépendante, la question de la légitimité de la rupture du lien colonial n'a plus de sens: l'indépendance est un fait, irréversible. Au contraire, ceux qui ont connu la guerre lisent les travaux historiques avec la question de l'appartenance partisane et classent les historiens dans des camps construits dans la contemporanéité du conflit: pro-indépendance, pro-Algérie française, libéraux, chrétiens progressistes, ou encore messaliste, frontiste... La réaction de certains officiers généraux, outrés que les historiens travaillent sur la répression, et en particulier la torture, révèle le hiatus existant entre une génération pour qui l'issue de la guerre est toujours débattue et une génération pour qui elle est incontestable ${ }^{18}$. Si elle est incontestable, tous les sujets sont ouverts, y compris les plus épineux, car, une fois la guerre terminée, il n’y a plus de camps en présence, d'ennemis ni d'adversaires qui pourraient profiter des faiblesses de l'autre, les exploiter pour l'emporter.

Reprocher l'absence de "l'autre camp » dans des travaux historiques menés aujourd'hui, prouve la persistance de la guerre dans les esprits de ceux qui l'ont vécue. Ainsi, pour prouver son objectivité quand il travaille sur la guerre d'indépendance algérienne, le jeune historien ne peut se contenter de l'exercice classique de critique des sources, de leur croisement, leur confrontation et leur analyse. Ce n'est pas sur ce terrain que son objectivité est mesurée. Il est attendu sur un terrain obsolète: celui de l'étude des deux camps, même si cela crée une absurdité sur le plan problématique. En ce qui concerne l'exercice de la justice française pendant cette guerre, que viendrait faire la justice de «l'autre camp » dans le sujet? Elles ne se répondaient pas, ne se combattaient pas l'une l'autre. La première est celle d'une République, qui se définit par son rapport à la loi, seule source de légitimité, y compris lorsqu'elle réprime une insurrection ou mène une guerre. La double conjonction de la guerre et du terrain colonial créait une exceptionnalité, intéressant, d'abord, les juristes ${ }^{19}$ : comment la République pouvait-elle gérer cette situation, sans contradiction avec ses grands principes? Quant à la « justice» du FLN, les guillemets s'imposent: a-t-elle existée ${ }^{20}$ ? Le FLN s'est construit dans la guerre, c'est-à-dire dans l'instabilité et la précarité, en l'absence de structure solidement

I8. En janvier 2002, 490 officiers généraux ont ainsi signé un manifeste pour justifier l'usage de la torture pendant la guerre d'Algérie, publié en introduction d'un Livre blanc de l'armée française en Algérie, aux éditions Contretemps.

19. Deux thèses ont ainsi été soutenues: A. Heymann (1972) et K. Vartanian (I995). L'Ecole nationale de la magistrature a aussi organisé un colloque en décembre 1995, Juger en Algérie, 1944-1962 (1997).

20. Voir ma contribution, «L'organisation judiciaire du FLN", dans C.R. Ageron (dir.) (1997 : 137-I49). 
établie, contrôlée d'en haut, et dans la rivalité pour le pouvoir conféré par la lutte pour l'indépendance ${ }^{21}$.

Létonnement de voir des femmes s'intéresser à la guerre d'indépendance algérienne relève aussi, en partie, d'un fossé générationnel car elle s'inscrit dans une représentation traditionnelle de la répartition des rôles entre sexes, la guerre, la violence et la répression étant du domaine masculin. L'évolution du statut des femmes depuis la fin des années 1960 produit ici ses effets: elle a décomplexé les jeunes nés avec elles et leur a ouvert la voie au CNRS ou à l'Université; elle leur a permis d'étudier le sang versé par les combats, la torture ou la guillotine. Après le travail de Djamila Amrane, les femmes dans cette guerre sont d'ailleurs devenues ellesmêmes objets d'étude ${ }^{22}$.

Le fait que la grille de lecture distinguant les camps en présence pendant la guerre d'indépendance algérienne n'a aucune pertinence pour disqualifier les travaux des jeunes historiens en les renvoyant à leur partialité, ne signifie pas, néanmoins, que les motivations politiques sont absentes de leurs démarches. Elles peuvent exister comme elles ont pu exister chez leurs aînés; simplement, elles ne sont plus les mêmes. Dans la génération accédant à la conscience politique dans les années I980, un faisceau de circonstances ramenait ceux qui s'intéressaient à l'histoire vers la guerre d'indépendance algérienne: élection à la présidence de la République de François Mitterrand, ministre de l'Intérieur et de la Justice dans les trois premières années de la guerre; exercice du pouvoir par la gauche pour la première fois depuis 1958; montée du Front national dirigé par un ancien partisan de l'Algérie française; réplique des organisations anti-racistes conférant à cette période un rôle pédagogique ${ }^{23} \ldots$ Cette guerre, dans son versant français, semblait détenir les clefs de cette période où les questions de nationalité, de racisme, des droits reconnus ou refusés à l'immigré venu des anciennes colonies et à ses descendants sur le sol de l'ancienne métropole, occupaient une grande partie du débat public ${ }^{24}$.

C'est la réflexion sur des questions appartenant aux décennies 1980-1990-2000 qui est en jeu dans les travaux des jeunes chercheurs d'aujourd'hui sur cette guerre, et non la réactivation d'un conflit appartenant aux générations passées. Cela explique l'attrait particulier exercé par les aspects purement français de ce conflit, ceux qui ont produit leurs effets jusqu’à marquer la société quarante ans plus tard et alimenter les débats en cours. Les institutions répressives, police, justice, armée,

2I. G. Meynier (2002).

22. Diane SAmbron prépare ainsi une thèse sur le statut des femmes algériennes pendant la guerre. Le $\mathrm{n}^{\circ} 75$ de Vingtième Siècle. Revue d'histoire, consacré à l'Histoire des femmes, histoire des genres fait aussi une place à cette guerre avec l'article de R. Branche (2OOI : I23-I32).

23. J'ai développé ce point de vue dans V. DuClert et alii (2002 : I35-I43).

24. Le sujet de thèse de Laure BLÉvis en témoigne directement, même s'il concerne la période antérieure à la guerre: «Citoyenneté et nationalité en Algérie coloniale. » 
retiennent ainsi l'attention ${ }^{25}$. Au contraire, les aspects algériens interpellent beaucoup moins cette génération, sauf ceux concernant les Algériens vivant en métropole $^{26}$.

"Léloignement » du "fait colonial et nous ", analysé par Daniel Rivet dès I992, ne pouvait que s'accroître avec le temps. Cependant, s'il permet effectivement de se délivrer " de la dialectique de la célébration et de la condamnation du fait colonial qui a si longtemps et si profondément biaisé l'écriture de son histoire ", il ne garantit pas une plus grande objectivité ${ }^{27}$. L'éloignement temporel ne signifie pas, en effet, une plus grande distance de l'historien avec l'événement qu'il étudie, au moment où il l'étudie. Le temps passant, c'est plutôt une subjectivité qui succède à une autre, d'où l'impression d'un renouveau de l'historiographie dès qu'une nouvelle génération, investissant l'étude d'une séquence historique, lui confere de nouveaux enjeux, ancrés dans les débats de son époque.

\section{L'OUVERTURE DES ARCHIVES MILITAIRES, UNE OPPORTUNITÉ}

endant dix ans, de 1992 à 2002, le Service historique de l'armée de terre a opté pour une politique d'ouverture de ses archives, avant de faire machine arrière face aux polémiques sur la torture qui ont mis en émoi les milieux militaires. C'est donc un regard rétrospectif qui est jeté sur cette période, pendant laquelle les documents du SHAT ont représenté la source privilégiée sur la guerre d'indépendance algérienne. Depuis, la publication de plusieurs articles relatifs aux fonds possédés par les dépôts d'archives civils a témoigné des efforts accomplis par diverses institutions ou responsables ${ }^{28}$.

L'ouverture des archives du SHAT a signifié l'établissement d'un inventaire de la série $\mathrm{IH}$, la disponibilité d'un personnel affecté à son traitement, ainsi que, dans un premier temps, la réservation d'une salle à sa consultation. Globalement, l'examen des demandes de dérogation a été libéral, avec des variations selon les sujets ou les responsables en place au moment de la demande. L'accès commode du château de Vincennes en proche banlieue parisienne a également favorisé l'utilisation de ces documents, la comparaison avec les difficultés matérielles actuelles pour travailler aux Archives nationales mettant clairement en relief l'importance de ce type

25. La police est étudiée par Emmanuel Blanchard et Rémi Valat en métropole, par Jean-Pierre PeYroulou en Algérie. Tramor Quémeneur, quant à lui, étudie les désertions, insoumissions et refus d'obéissance de la part des soldats français.

26. Voir L. Pitti (2002) et L. Amiri (200I et 2002).

27. D. Rivet (I992 : I28).

28. F. Banat-Berger et C. Noulet (2000 : 327-35I) pour les Archives de la Justice et le CHAN; A. Goudail, (2000 : 19-40), consacré au Centre des archives d'Outre-mer; A. Callu et P. Gillet (2002 : 47-63) ajoutent peu de choses aux références présentées par Françoise Banat-Berger et Christèle Noulet. 
de considérations dans le travail historique. L'ensemble a créé des conditions propices à l'utilisation des archives militaires pour renouveler l'écriture de l'histoire.

Les raisons de cette ouverture ont été multiples, au premier rang desquelles les questions matérielles ne doivent pas être négligées, la main-d'œuvre des appelés ayant amplement contribué au fonctionnement du SHAT. Politiquement, ensuite, le SHAT a fait le pari que l'ouverture des archives valait mieux qu'une attitude frileuse, pour des raisons d'image mais aussi dans l'intérêt propre de l'armée. L'accès privilégié à des documents militaires aurait pu donner naissance à des travaux reprenant le point de vue militaire, le défendant, au besoin, contre des travaux plus critiques. D'une façon générale, l'ouverture des archives n'est pas forcément risquée, elle peut aussi présenter des avantages: si les documents peuvent étayer des points de vue mettant l'armée en cause, ils peuvent aussi fournir des arguments à ses défenseurs. La meilleure illustration de cet intérêt bien compris, à la fois en termes politiques et en termes de communication, a été la publication d'une dépêche AFP annonçant l'achèvement de l'inventaire des archives militaires concernant cette guerre, le 30 octobre 200I, alors que l'affaire Aussaresses venait de défrayer la chronique. Le général Berlaud, alors chef du SHAT, déclarait qu’il serait « dans les jours qui viennent, à la disposition des chercheurs comme de toute personne intéressée. "

Logiquement, avec ces facilités offertes aux historiens, l'histoire militaire de la guerre d'indépendance algérienne a connu une expansion, que le colloque organisé par Maurice Vaïsse et Jean-Charles Jauffret en mai 2000 a rendu visible ${ }^{29}$. Plusieurs thèses et publications témoignent également de l'éclosion de travaux dans ce domaine, où cette guerre s'intègre parfois à des perspectives de longue durée ${ }^{30}$. Le développement de cette histoire militaire répond à une double et légitime préoccupation. D'abord, la revalorisation des aspects militaires admet cette guerre comme telle, alors qu'elle a été si longtemps niée. Les travaux historiques mettant l'accent sur les combats, les stratégies, le monde militaire... valent reconnaissance de ce conflit pour ce qu'il a été. Cette histoire rencontre ensuite l'intérêt de l'opinion car, dans la société française d'aujourd'hui, plusieurs centaines de milliers d'anciens d'Algérie, appelés, rappelés ou engagés, constituent un public pour ce type de récit ou d'analyse, dans lesquels ils peuvent retrouver une partie de leur vécu personnel. L'histoire militaire trouve aisément ses repères dans les représentations forgées par l'expérience directe qu'ils ont eue du conflit. Le vocabulaire, notamment, élément structurant du groupe combattant, leur est familier ${ }^{31}$. Un des revers de la consultation privilégiée des documents du SHAT est d'ailleurs de plon-

29. J.-C. Jauffret et M. VÄ̈sSe (200I). Voir aussi le colloque d'octobre 2002, J.-C. JaUfFret (dir.) (2003). 30. Cf. J. Valette (dir.) (2003) et les thèses de X. Boniface (200I), F. Médard (2002), P. et M.C. VIllatoux (2002). La connaissance du monde combattant de l'ALN reste en souffrance, Dalila AïTEL-Djoudi, isolée dans ce domaine, étudiant l' "Image des combattants français vue par l'ALN, I954-I962".

31. Cf. J.-C. JaufFret (2002: 37-55). 
ger les chercheurs dans l'univers militaire, et, en l'absence d'autres sources pour le relativiser, de le restituer sans nuance ou avec une distance insuffisante. Les historiens algériens se sont ainsi insurgés contre l'emploi de termes tels que "rebelles ", "fellaghas ", voire même " fell ", par certains historiens français qui les reprenaient à leur compte, après s'y être accoutumés à force de les lire dans les documents produits par l'armée de terre au moment même des faits, documents devenus archives sur lesquelles ils fondent leur travail.

Cependant, l'histoire militaire, à elle seule, ne livre pas les clefs du conflit car cette guerre a été une guerre sans bataille décisive, sans victoire, ni défaite des uns ou des autres. Aucun Stalingrad ou Dien Bien Phu n'est venu scander sa chronologie. Son évolution, sa conduite, son issue même, ne trouvent guère d'explication dans les opérations militaires, au point que la thèse d'une victoire de l'armée, trahie par le pouvoir politique qui a négocié l'indépendance, a été défendue. En réalité, cette guerre s'est jouée sur d'autres terrains, d'autant plus que le FLN a usé de l'arme terroriste, de l'offensive diplomatique et du rapport de force politique. L'histoire politique de la France, du mouvement national algérien ou encore des relations internationales apparaît ainsi comme la plus pertinente pour accéder à la compréhension de ce conflit. C'est pourquoi l'historiographie reste marquée par un fort déséquilibre au détriment de l'histoire économique et sociale, et en faveur de l'histoire politique.

L'ouverture des archives, les facilités offertes ou les difficultés opposées à leur consultation ont suscité maints débats ${ }^{32}$. Plus que cette question cependant, celle de l'influence des archives utilisées sur la construction même des problématiques par les historiens devrait interpeller. La question n'est pas seulement de savoir avec quelles sources travaillent les historiens, mais plutôt de se demander à quelle histoire donnent naissance les sources disponibles.

\section{Quelles SOURCES POUR QUELLE HISTOIRE?}

Cette question mérite d'autant plus de retenir l'attention que les archives militaires n’ont pas été utilisées pour étudier les seuls aspects militaires du conflit. L'accès privilégié à ces archives a alors orienté la définition et le traitement des sujets. Dans le cas des institutions judiciaires, par exemple, qu'aurait été le traitement du sujet sans les archives du ministère de la Justice? Le SHAT recèle en effet une très grande masse de documents relatifs à la législation, aux condamnations, au fonctionnement des tribunaux, à l'attitude des magistrats... Cette documentation, très riche, aurait pu se suffire à elle-même, sauf que des pans entiers du sujet auraient échappé à la connaissance, très peu de documents conservés au SHAT

32. En désaccord à propos des archives de la préfecture de police, cf. C. Liauzu (200I : 53-56) et J.-M. BERLIÈre (200I : 57-68). 
émanant des ministres ou des magistrats eux-mêmes. Ils n'étaient rencontrés que par l'intermédiaire des documents de l'armée. Or, ces documents livrent la perception des magistrats et de la justice par les militaires, la représentation qu'ils s'en font. Le sujet, apprécié par la médiation des militaires, devenait plutôt "l'armée et la justice » ou « les militaires et les magistrats. » Il était nécessaire d'accéder aux archives du ministère de la Justice pour accéder aux pans civils et politiques du sujet, pour voir s'exprimer, s'animer eux-mêmes, sans intermédiaire, les gardes des Sceaux et les magistrats. Il était possible alors, de relativiser et critiquer la version qui émergeait des archives militaires. La prise de distance avec une source unique d'archives est un exercice classique pour l'historien. Elle pêche néanmoins dans le cas de la guerre d'indépendance algérienne: elle devrait s'appliquer à l'orientation de la problématique par les archives militaires.

En outre, l'usage exclusif des archives militaires impose une restriction spatiale et chronologique à la recherche. En effet, l'armée s'étant massivement déployée sur le territoire algérien, ses documents sont riches pour celui-ci mais muets pour la métropole. Ce déséquilibre ne porte pas préjudice au traitement des sujets de recherche, car les deux territoires présentent des configurations réellement et suffisamment différentes pour qu'un sujet soit conçu dans l'un ou l'autre de ces espaces seulement. Envisager Algérie et métropole ensemble, dans une seule étude, serait même aberrant dans de nombreux cas. Ce déséquilibre se répercute cependant sur l'avancée des connaissances entre les deux territoires: les progrès récents de l'historiographie ont concerné, d'abord et avant tout, la guerre en Algérie et non en métropole. Celle-ci est restée le lieu privilégié de l'étude de l'opinion ou des événements parisiens considérés comme nationaux. Le surinvestissement sur la manifestation du 17 octobre 196I et sa répression pourrait ainsi masquer l'ignorance sur le reste de la période et en dehors de la région parisienne ${ }^{33}$.

La restriction chronologique est plus gênante car elle occulte l'avant-I954. Le SHAT possède évidemment des archives sur la période antérieure au déclenchement de la guerre, mais en quantité moins importante que pour la guerre ellemême ${ }^{34}$. L'histoire construite sur ces seules archives aboutit à une chronologie reflétant, en réalité, l'investissement de l'armée dans le domaine étudié. Le cas de l'action sanitaire et sociale est particulièrement flagrant: tant que l'armée ne l'avait pas pris en charge, en effet, elle ne pouvait produire des documents à son sujet; les archives militaires permettent, par conséquent, de savoir quand l'armée s'y est investie et, à partir de cette date, d'étudier son action. Mais elles ne permettent pas de situer cette action dans la continuité, ou dans la rupture, avec celle des autorités civiles. Le risque existe alors de reproduire l'argumentation des milieux mili-

33. C'est pourquoi l'IHTP a lancé un programme de recherche sur la guerre d'Algérie en métropole, réalisé par le réseau des correspondants départementaux.

34. Voir les deux tomes de documents publiés sous la direction de J.-C. JaUfFret (I990-I998) qui s’arrêtent en 1954 . 
taires, consistant à expliquer que l'armée est venue se substituer à des autorités civiles absentes ou défaillantes. Les archives militaires n'éclairent pas la question de la rupture de 1954: que change le déclenchement de la guerre, dans les autres domaines que le domaine militaire ? Il faudrait disposer d'une masse documentaire aussi importante sur la période antérieure à 1954 pour pouvoir répondre.

Enfin, l'analyse du pouvoir pendant la guerre d'indépendance algérienne est à l'heure actuelle surdéterminée par la documentation militaire. La réflexion et les débats reposent en effet sur l'idée d'un pouvoir de l'armée fort, opposé au politique et le dominant. Les archives du SHAT donnent effectivement l'impression que l'armée est toute puissante, que son pouvoir connait une croissance continue pendant le conflit et surtout, que l'armée s'oppose au politique, qu'elle cherche à subordonner. Dans le cas de la justice, cette version des événements conduirait à penser que toutes les réformes ont été voulues par l'armée, qu'elles répondaient à ses vœux. Le pouvoir politique n'aurait fait que se plier aux revendications des militaires qui, satisfaits de voir leurs pressions aboutir, en demandaient toujours plus. Exposée ici de façon caricaturale, cette version des rapports entre politique et militaire est celle qui ressort de la consultation unique des documents de l'armée de terre.

Or, si le pouvoir acquis par l'armée est réel et important, ses manifestations dans les documents relèvent aussi, parfois, d'un fantasme de l'auteur sur ce que l'armée peut se permettre. C'est le cas de la note envoyée par le colonel Argoud au ministre de la Justice Edmond Michelet en novembre 1959: son auteur demande notamment que le commandement puisse interférer dans l'exercice du droit de grâce présidentiel ${ }^{35}$. Cette note est significative de la représentation que certains militaires se faisaient de leur propre pouvoir: le colonel Argoud s'est senti assez sûr de lui pour aller jusqu'à proposer une intervention dans un domaine étroitement réservé au président de la République, à la fois par la tradition régalienne et par la conception du pouvoir présidentiel qui animait le général de Gaulle. Sur ce point précis, le colonel Argoud a surestimé les potentialités d'accroissement du pouvoir de l'armée. En dehors de cet exemple frappant, quantité d'autres documents livrent une version plus subtile de cette surestimation, qui peut piéger l'historien et aboutir à une histoire dans laquelle le militaire, tout puissant, est présenté comme le seul acteur décisif de cette guerre.

Par ailleurs, les documents de l'armée incitent à raisonner sur une opposition entre civils et militaires. Schématiquement, les années 1954-1962 se diviseraient entre une $\mathrm{IV}^{\mathrm{e}}$ République, qui aurait laissé s'accroître les pouvoirs de l'armée, et la suivante qui les aurait progressivement réduits au profit d'une restauration des autorités civiles. Cette présentation souffre d'une conception erronée des mondes civil et militaire: forment-ils chacun des camps homogènes, irréductiblement

35. Cette note a été présentée et publiée par P. VIDAL-NAQUET dès 1962 aux éditions de Minuit (2002 pour la réédition: 268-274). 
opposés l'un à l'autre? Cette vision du monde séparant strictement civils et militaires appartient à l'armée et d'autres oppositions peuvent se révéler plus pertinentes pour l'historien. Opposer, par exemple, Paris et Alger, où des civils ont fait corps avec le commandement. Le cas de Robert Lacoste, ministre résident de 1956 à 1958 , en est le plus révélateur: jamais en opposition avec l'armée, il n'était pas non plus en état de soumission; il était lui-même sincèrement convaincu que la situation exigeait un accroissement des pouvoirs répressifs de l'armée, grâce à un transfert des pouvoirs des autorités civiles vers les militaires.

Transcendant cette opposition entre Alger et Paris, deux conceptions de la politique de répression à mettre en œuvre en Algérie se sont aussi opposées, à l'intérieur de l'armée et au sein des autorités civiles: la première considérait que l'armée devait avoir tous les moyens d'agir, tout contrôle étant assimilé à un handicap dans l'action; la seconde considérait comme nécessaire d'encadrer l'action des militaires. À Paris, des hommes politiques, comme Maurice Bourgès-Maunoury, ont défendu d'eux-mêmes la première option, sans être dominés ni manipulés par les militaires; à Alger, au contraire, certains responsables civils, comme Paul Teitgen, secrétaire général de la préfecture de police, ou Jean Reliquet, procureur général, ont pensé qu'il fallait mesurer les pouvoirs accordés aux militaires.

L'opposition entre civils et militaires reproduit ainsi trop facilement une vision du monde appartenant à l'univers militaire. C'est cette vision qui a été activée pendant les polémiques des années 2000-2002 sur la pratique de la torture par l'armée française pendant la guerre d'indépendance algérienne. Les militaires mis en accusation ont ainsi rejeté les responsabilités sur le pouvoir politique, comme si civils et militaires formaient deux groupes sans divisions internes et clairement constitués, sans passerelle ni lien de l'un à l'autre. En réalité, les oppositions entre civils et militaires, entre Paris et Alger, entre des conceptions sur l'aménagement du système répressif, ont toutes joué: elles se sont combinées, recoupées, succédé dans le temps.

Ainsi, l'expérience d'un travail de recherche mené en grande partie sur les archives militaires oriente la réflexion vers l'influence exercée par la consultation privilégiée d'un fonds d'archives sur l'écriture de l'histoire. Cette question est pourtant absente des débats sur les relations entre archives et histoire, la question de l'accès aux documents occupant l'essentiel des débats.

\section{Traverser la Méditerranée}

L'émergence d'une nouvelle génération de chercheurs, ainsi que l'utilisation des archives militaires pour écrire l'histoire de cette guerre s'inscrivent dans un cadre proprement français alors que cette histoire est une histoire partagée. C'est une histoire, cependant, dont le partage avec l'autre est plus douloureux qu'entre vainqueur et vaincu, qu'entre anciens adversaires ayant conjointement décidé de 
mettre un terme aux combats par le cessez-le-feu. Il s'agit en effet de partager I30 ans d'histoire faits de domination, conflits latents et récurrents mais aussi d'une acculturation source de culpabilité ${ }^{36}$.

Aujourd'hui, cependant, les problèmes posés par la question du travail de recherche en Algérie ne sont pas tant ceux du travail dans une ancienne colonie, que ceux du travail dans un pays non démocratique et en proie à une guerre civile. Les questions administratives ne sont pas les plus difficiles à résoudre: l'obtention d'un visa, les formalités d'accès aux bibliothèques et centres d'archives sur place ne posent guère de problèmes et ouvrent aux chercheurs " un terrain algérien ", riche et prometteur ${ }^{37}$. Le reflux de l'insécurité dans les grandes villes permet également d'envisager, plus facilement qu'il y a quelques années, de traverser la Méditerranée. Si tous les sujets ne nécessitent pas l'exploration d'un " terrain algérien ", surtout lorsqu'il s'agit d'étudier la guerre dans son versant français ou métropolitain, les échanges avec les chercheurs algériens paraissent, eux, indispensables.

Dans un pays où l'écriture de l'histoire a rempli une fonction de légitimation du pouvoir, l'État a tenté de maîtriser son élaboration par les centres de recherche et sa diffusion tant par l'enseignement que par la publication d'ouvrages ${ }^{38}$. Après les événements d'octobre 1988, le régime avait entrepris un processus de démocratisation, stoppé par le coup d'État de janvier 1992 et la guerre dans laquelle le pays est toujours plongé. Ces quatre années ont cependant permis l'émergence d'institutions nouvelles, les Fondations, jouant un rôle central dans le domaine historique.

Dotées d'un régime juridique issu de la loi autorisant les associations en 1989 , elles témoignent de l'émergence d'une société civile algérienne, dans une forme d'organisation indépendante du pouvoir et ont investi des domaines très divers, de l'humanitaire au financement de la recherche scientifique. Attachées à l'exercice de célébration mémorielle, comme la Fondation du 8 mai 1945, la Fondation Boudiaf ou la Fondation El Mokrani, elles ont aussi, logiquement, occupé le terrain historique en organisant des colloques, rencontres, tables rondes, d'autant qu'elles disposent de moyens financiers supérieurs à ceux des universités.

Difficile cependant de se repérer dans le dédale de réseaux que semble former la société algérienne: par leurs financements, par les hommes qui les dirigent, les Fondations sont aujourd'hui souvent identifiées comme appartenant à tel ou tel réseau contre tel ou tel autre. Les réticences à répondre aux invitations venues d'Algérie et lancées à de jeunes historiens qui n'y ont aucune relation personnelle

36. "L'acculturation à la française signifiait d'un côté la possibilité la possibilité de humer des vents frais de l'extérieur, voire d'en désirer la caresse. Le souffle de ces vents était employé à faire vaciller les étouffoirs communautaires du conservatisme algérien. D'un autre côté, cette acculturation était plus ou moins ressentie comme une trahison au regard des normes communautaires ", écrit G. MeYNIER (2002:47).

37. R. Bader, D. Guignard et A. Kudo ont témoigné d'une mission prospective qu'ils ont effectuée en Algérie en juillet 2002 (2003: IIO-II2).

38. Voir B. STORA (2000: IO9-II8). 
s'expliquent ainsi. Il ne s'agit pas d'un mépris perpétuant le regard hautain et dédaigneux jeté sur l'ancienne colonie; il ne s'agit pas non plus de la négligence pour un " terrain algérien " considéré a priori comme pauvre et inaccessible; il s'agit encore moins de dénigrer les travaux et les publications des Algériens s'intéressant à leur guerre d'indépendance, qui se sont multipliés dans la décennie passée $^{39}$. Il s’agit de savoir qui invite et pourquoi.

La question se pose pour toute sollicitation du chercheur sur un objet d'histoire aux enjeux politiques forts, que ce soit en Algérie ou ailleurs, car il n’y a évidemment pas qu'en Algérie que "le présent commande au passé ${ }^{40}$. Les polémiques autour de la torture pendant la guerre d'indépendance algérienne, qui ont surgi en France, en 2000-200I, le prouvent. Cependant, pour un jeune historien français, s'il est possible de se repérer dans le débat public français, d'en discerner les arrièrepensées, la tâche est plus complexe dans l'Algérie d'aujourd'hui, surtout lorsqu'elle est, pour lui, une société inconnue.

Les enjeux sont, en outre, d'une autre gravité: comment un historien, qui a fait le choix, précisément, de travailler sur le système répressif construit par la France contre les indépendantistes algériens, peut-il ignorer les massacres, les exécutions, les disparitions, la torture contemporains ? Souvent, la méconnaissance du «terrain algérien » est reprochée aux jeunes chercheurs comme une lacune témoignant de la persistance de l'ignorance de l'Autre, comme au temps où l'histoire des colonies appartenait au seul colonisateur. Mais le temps des colonies appartient au passé. Ce sont d'autres problèmes que soulève la question des échanges avec l'Algérie. Travailler avec l'ancien adversaire n'est pas aussi facile pour la guerre d'indépendance algérienne que pour la Première ou la Seconde Guerre mondiales, précisément parce qu'il ne s'agit pas de travailler avec l'ancien adversaire, mais avec ce qu'il est devenu aujourd'hui. Il ne s'agit pas seulement, en effet, de travailler avec un pays anciennement colonisé par la France mais aussi de travailler avec un pays non démocratique et en guerre. Ces données sont pour l'historien, aujourd'hui, fondamentales.

Cette tentative pour décoder l'évolution récente de l'historiographie française aboutit finalement à définir les contours d'une démarche épistémologique qui pourraient contribuer à renouveler les connaissances et l'approche de cette période. Le recours systématique à l'expression de " guerre d'indépendance algérienne ", audelà des catégories partiales, "guerre d'Algérie » ou "Révolution », "guerre de libération ", utilisées de part et d'autre, marque une étape dans la construction de cette séquence du passé comme catégorie historique, que les historiens peuvent choisir de nommer selon sa nature: une guerre dans laquelle s'est construite l'Algérie d'au- 
jourd'hui. Une diversification des sources, mettant l'accent sur le civil et le politique, permettrait aussi, certainement, de renouveler les représentations collectives de ce conflit, trop souvent ramené à sa version militaire.

Le travail avec l'Algérie, enfin, s'impose comme une nécessité, mais en posant des problèmes politiques et moraux dépassant la seule question du travail avec et sur une ancienne colonie française, alors que, pendant longtemps, c'est ainsi que les difficultés de l'échange avec le sud de la Méditerranée étaient présentées. Mais n'est-ce pas, finalement, le défi de tout historien travaillant sur l'histoire dite « du temps présent " ?

\section{A Bstract}

This article aims at going over the writing, in the context of 1990-2000, of the history of the war called the "Algerian War". Those years saw the coming of age of a new generation of historians, who have not lived through this war and who actually reinvest in it political agendas of their own time. The opening up of archives from the Army Historical Service (Service historique de l'armée de terre : SHAT) between 1992 and 2000 has also influenced the historiography, multiplying studies on the military aspects of the war. It also has produced a history reflecting the military point of view that other sources ought to correct. And last scientific exchanges with Algeria, for a long time moulded by the former colonial relations, are now also disturbed by the absence of democracy and the war going on in that country. These conditions call for historians working on the Algerian independence war to define anew their historical approach.

\section{Bвйоскиние}

Ageron C.R. (dir.), La guerre d'Algérie et les Algériens, Paris, IHTP/Armand Colin, 1997. Ageron C.R., Les Algériens musulmans et la France de I87i à I9I9, Paris, PUF, I97I.

Amiri L., I7 octobre I96I. Analyse des faits à travers les archives de la Fédération de France du FLN, celles de la Préfecture de police de Paris et de la CIMADE, mémoire de maîtrise en histoire à Paris VIII, 200I.

Amiri L., L'émigration algérienne dans le département de la Seine: entre encadrement, contrôle et répression. La Préfecture de police et le FLN (1958-1962), mémoire de DEA en histoire de l'IEP de Paris, 2002.

Amrane D., Les femmes algériennes dans la guerre, Paris, Plon, I99I.

Banat-Berger F. et Noulet C., "Les sources de la guerre d'Algérie aux Archives nationales ", in Revue française d'histoire d'outre-mer, $\mathrm{n}^{\circ} 328-329,2^{\circ}$ semestre 2000, p. 327-35I.

Bader R., Guignard D., Kudo A., "Un terrain algérien pour la recherche ", in Vingtième Siècle. Revue d'histoire, $\mathrm{n}^{\circ}$ 77, janvier-mars 2003, p. IIO-II2.

BECKER J.-J., " Du contemporain à l'historien. Autour de l'histoire du temps présent ", in S. Audouin-Rouzeau et al (dir.), La politique et la guerre. Pour comprendre le XX' siècle européen, Paris, Éditions Agnès Viénot/Noésis, 2002, p. 35-46. 
BÉdarida F. et Fouilloux E. (dir.), Les chrétiens dans la guerre d'Algérie, dans Cahiers de l'Institut d'histoire du temps présent, $\mathrm{n}^{\circ}$ 9, octobre $\mathrm{I} 988$.

BERLIÈre J.-M., "Archives de police/historiens policés? ", in Revue d'histoire moderne et contemporaine, $\mathrm{n}^{\circ}$ 48-4 bis, supplément 200I, p. 57-68.

Boniface X., L'aumônerie militaire française (I9I4-I962), Paris, Le Cerf, $200 \mathrm{I}$.

Bonnaud R., "Torture: l'histoire et la mémoire ", in Les Inrockuptibles, 19-25 décembre 2000, p. 70-7I.

Branche R., "Des viols pendant la guerre d'Algérie ", Vingtième Siècle. Revue d'histoire, $\mathrm{n}^{\circ} 75$, juillet-septembre 200I, p. I23-I32.

Branche R., La torture et l'armée pendant la guerre d'Algérie, Paris, Gallimard, coll. « La suite des temps ", 200I.

Callu A. et Gillet P., "Écrire les traumatismes de la guerre d'Algérie aux Archives nationales ", in Histoire et Archives n ${ }^{\circ}$ II, janvier-juin 2002, p. 47-63.

CARLIER O., Entre nation et djihad. Histoire sociale des radicalismes algériens, Paris, Presses de Sciences Po, I995.

Droz B. et Lever E., Histoire de la guerre d'Algérie, Paris, Seuil, coll. " Points Histoire ", 1982.

Frémeaux J., Les bureaux arabes dans la province d'Alger (I844-I856), Paris, Denoël, I993.

Goudail A., "Les sources françaises de la guerre d'Algérie ", in D. Lefeuvre et A.-M. Pathé (dir.), La guerre d'Algérie au miroir des décolonisations françaises, Paris, SFHOM, 2000, p. 19-40.

Hamon H. et Rotman P., Les porteurs de valise. La résistance française à la guerre d'Algérie, Paris, Seuil, coll. " Points histoire ", I982 (rééd.).

Heymann A., Les libertés publiques et la guerre d'Algérie, Paris, LGDJ, 1972.

Jaufretet J.-C. (dir.), Des hommes et des femmes en guerre d'Algérie, Paris, Autrement, coll. " Mémoires " n 97, 2003.

JAUfFret J.-C., "Entendre et enseigner l'expérience du combattant français de la guerre d'Algérie ", in D. Borne et al (dir.), Apprendre et enseigner la guerre d'Algérie et le Maghreb contemporain, Buc, CRDP de l'Académie de Versailles, 2002, p. 37-55.

JAUfFret J.-C., La guerre d'Algérie par les documents, Tome I, L'avertissement (février I943mars 1946) et tome II, Les portes de la guerre (avril 1946-décembre 1954), Vincennes, SHAT, I990-1998.

Jauffret J.-C. et VAïsse M. (dir.), Militaires et guérilla dans la guerre d'Algérie, Bruxelles, Complexe, 200 I.

Juger en Algérie, 1944-I962, dans Le Genre humain n 32, septembre 1997.

Lefeuvre D., Chère Algérie, Paris, SFHOM, 1997.

Liauzu C., "Interrogations sur l'histoire française de la colonisation ", in Genèses n 46 , mars 2002, p. 44-59.

Liauzu C., " Notes sur les archives de la guerre d'Algérie ", in Revue d'histoire moderne et contemporaine, $\mathrm{n}^{\circ}$ 48-4 bis, supplément 200I, p. 53-56.

Manceron G. et Remaoun H., D'une rive à l'autre: la guerre d'Algérie de la mémoire à l'histoire, Paris, Syros, 1993.

Mauss-Copeaux C., Appelés en Algérie. La parole confisquée, Paris, Hachette Littératures, 1998.

MéDARd F., Technique et logistique en guerre d'Algérie, Paris, Panazol/Lavauzelle, 2002.

Meynier G., Histoire intérieure du FLN, Paris, Fayard, 2002.

Meynier G., L’Algérie révélée. La guerre de I9I4-I9I8 et le premier quart du vingtième siècle, Paris, Droz, I98I.

Pervillé G., Les étudiants algériens de l'université française (I882-I962), Paris, CNRS, I984. 
PIтtт L., Ouvriers algériens à Renault-Billancourt de la guerre d'Algérie aux grèves d'OS des années 1970. Contribution à l'histoire sociale et politique des ouvriers étrangers en France, thèse de doctorat en histoire de Paris VIII, 2002.

Rey-Goldzeiguer A., Le royaume arabe. La politique algérienne de Napoléon III, Alger, OPU, 1977.

Rioux J.-P. (dir.), La guerre d'Algérie et les Algériens, Paris, Fayard, I990.

Rioux J.-P. et Sirinelli J.-F. (dir.), Les intellectuels et la guerre d'Algérie, Bruxelles, Complexe, I99I.

RIvet D., "Le fait colonial et nous. Histoire d'un éloignement ", in Vingtième Siècle. Revue d'histoire, $\mathrm{n}^{\circ}$ 33, janvier-mars 1992, p. I27-I38.

Rousso H., Vichy. L'événement, la mémoire, l'histoire, Paris, Gallimard, coll. "Folio Histoire ", 200I.

Stora B., Histoire de la guerre d'Algérie, Paris, La Découverte, coll. "Repères ", I993.

Stora B., Ils venaient d'Algérie, Paris, Fayard, 1992.

Stora B., "Maroc-Algérie. Retour du passé et écriture de l'histoire », in Vingtième Siècle. Revue d'histoire, $\mathrm{n}^{\circ}$ 68, octobre-décembre 2000, p. I09-II8.

Stora B., Messali Hadj (I898-1974), pionnier du nationalisme algérien, Paris, L'Harmattan, I986

Thénault S., Une drôle de justice. Les magistrats dans la guerre d'Algérie, Paris, La Découverte, coll. "L'espace de l'histoire ", $200 \mathrm{I}$.

Thénault S., " De la guerre d'Algérie dans les argumentaires FN/anti-FN » in V. Duclert et al (dir.), Il s'est passé quelque chose... le 21 avril 2002, Paris, Denoël, 2002, p. I35-I43.

Valette J. (dir.), "Aspects militaires de la guerre d'Algérie ", Guerres mondiales et conflits contemporains, $\mathrm{n}^{\circ} 208$, janvier-mars 2003.

VARTANian K., Le droit et la guerre d'Algérie. Réflexion sur l'exceptionnalité, thèse de doctorat en droit de Paris X, 1995.

Vidal-Naquet P., Face à la raison d'État, Paris, La Découverte, I989.

VIDAL-NaQuet P., La raison d'État, Paris, La Découverte, 2002 (rééd.).

Villatoux P. et M.C., La guerre et l'action psychologique en France (I945-1960), thèse de doctorat en histoire en Sorbonne, 2002. 